\title{
Práticas culturais e incidência de manchas foliares em trigo ${ }^{(1)}$
}

\author{
Ariano Moraes Prestes ${ }^{(2)}$, Henrique Pereira dos Santos ${ }^{(2)}$ e Erlei Melo Reis(3)
}

\begin{abstract}
Resumo - Em experimento de campo, foram avaliados os efeitos de práticas culturais na incidência de manchas foliares, causadas por Drechslera tritici-repentis, Bipolaris sorokiniana e Stagonospora nodorum, e no rendimento de grãos de trigo. Foram comparados quatro sistemas de manejo de solo (plantio direto, cultivo mínimo, preparo convencional do solo com arado de discos + grade de discos e preparo convencional do solo com arado de aivecas + grade de discos) e três sistemas de rotação de culturas (trigo/soja; trigo/soja e ervilhaca/milho ou sorgo; e trigo/soja, aveia-preta ou aveia-branca/ soja e ervilhaca/milho ou sorgo). O delineamento experimental foi em blocos ao acaso, com parcelas subdivididas e três repetições. A parcela principal foi constituída pelos sistemas de manejo de solo, e as subparcelas, pelos sistemas de rotação de culturas. A incidência de manchas foliares de trigo foi mais elevada sob monocultura e sob plantio direto do que nos sistemas de rotação de culturas. A rotação de culturas viabilizou o plantio direto, em relação ao controle de manchas foliares, e garantiu a estabilidade do rendimento de grãos de trigo.
\end{abstract}

Termos para indexação: manejo do solo, sistema de cultivo, doenças das plantas, grãos, rendimento.

\section{Effect of cultural practices on the incidence of leaf blotches of wheat}

\begin{abstract}
The effects of cultural practices on leaf blotches of wheat, caused by Drechslera tritici-repentis, Bipolaris sorokiniana and Stagonospora nodorum, and on grain yield were assessed in field experiments at Embrapa-CNPT, in Passo Fundo, RS, Brazil. Four soil management systems (no-tillage, minimum tillage, conventional tillage using a disk plow plus disk harrow, and tillage using a moldboard plow plus disk harrow) and three crop rotation systems [system I (wheat/soybean), system II (wheat/soybean and common vetch/corn or sorghum), and system III (wheat/soybean, black oats or white oats/soybean, and common vetch/corn or sorghum)] were compared. A randomized block experimental design, with split-plots and three replicates, was used. Leaf blotch incidence was higher under monoculture and no-till than under crop rotation. Crop rotation under no-tillage controlled leaf blotch diseases and maintained yield stability of wheat.
\end{abstract}

Index terms: soil management, cropping systems, plant diseases, grain, yields.

\section{Introdução}

Na década de 70, demonstrou-se experimentalmente, na Embrapa-Centro Nacional de Pesquisa de Trigo, que os rendimentos de cereais de inverno poderiam ser triplicados através do cultivo num sistema diferente da sucessão trigo-soja, considerado na época como rotação de culturas (Reis, 1998).

\footnotetext{
(1) Aceito para publicação em 13 de junho de 2001.

(2) Embrapa-Centro Nacional de Pesquisa de Trigo, Caixa Postal 451, CEP 99001-970 Passo Fundo, RS. Bolsista do CNPq. E-mail: ariano@cnpt.embrapa.br, hpsantos@cnpt.embrapa.br

(3)Universidade de Passo Fundo, Fac. de Agronomia e Medicina Veterinária, Caixa Postal 566, CEP 99001-970 Passo Fundo, RS. E-mail: erleimelo@uol.com.br
}

A rotação de culturas constituindo o sistema plantio direto mantém, na superfície do solo, os restos culturais das diferentes espécies usadas no sistema (Santos et al., 1993). Entretanto, esses restos culturais podem multiplicar os patógenos agentes causais de doenças de cereais de inverno, como por exemplo os parasitas emibiotróficos, que causam podridões radiculares e manchas foliares.

As principais manchas foliares do trigo, no Sul do Brasil, são a mancha amarela da folha, causada por Drechslera tritici-repentis (Died), a mancha marrom, causada por Bipolaris sorokiniana (Sacc.), e a septoriose, causada por Stagonospora nodorum (Berk) (Reis, 1994).

As sementes infectadas e os restos culturais constituem as principais fontes de inóculo primário para 
os fungos causadores de manchas foliares em trigo (Rees \& Platz, 1980; Wiese, 1987; Reis et al., 1992). A mancha amarela da folha de trigo é de ocorrência mundial, sendo a mancha foliar mais freqüente e mais intensa nas lavouras em que o trigo é cultivado em monocultura e em sistema plantio direto (Rees \& Platz, 1980; Sutton \& Vyn, 1990; Reis \& Casa, 1996). Bipolaris sorokiniana não é considerado patógeno severo à cultura de trigo nas regiões mais frias do Brasil, porém o agente patogênico dessa doença é o fungo mais freqüente associado à semente de trigo (Reis et al., 1997).

Ruedell (1995), em trabalhos realizados na região de Cruz Alta, RS, com sistemas de manejo de solo, observou que entre várias culturas, o trigo foi a espécie que demonstrou menor resposta quanto ao rendimento de grãos. Contudo, na maioria dos anos do período estudado, o trigo cultivado em plantio direto apresentou rendimento de grãos mais elevado do que no sistema convencional de preparo do solo. De acordo com Ruedell (1995) e com Santos et al. (1996), a rotação de culturas permite manter e estabilizar o rendimento de grãos pela diversificação de espécies. Isso permite que os resíduos vegetais sejam decompostos a tempo de eliminar o inóculo da área de cultivo (Reis, 1991). Entretanto, a mineralização de resíduos vegetais, para completa eliminação de patógenos do sistema radicular (podridão-comum e mal-do-pé), pode levar de 12 a 16 meses (Reis \& Santos, 1993).

No Estado do Paraná, Santos et al. (1996) observaram maior rendimento de grãos de trigo nos sistemas com rotação de culturas e sob plantio dire- to do que sob monocultura desse cereal. Na Inglaterra, Slope et al. (1973) também verificaram rendimento de grãos de trigo mais elevado com dois invernos de rotação, em relação à monocultura dessa gramínea, enquanto no Canadá, Sturz \& Bernier (1989) relataram maior rendimento de grãos com um inverno sem esse cereal, alternado com colza ou com linho, em comparação com a monocultura de trigo.

O presente trabalho teve por objetivo avaliar os efeitos de práticas culturais sobre a incidência de manchas foliares e sobre o rendimento de grãos.

\section{Material e Métodos}

O ensaio foi conduzido na Embrapa-Centro Nacional de Pesquisa de Trigo, no Município de Passo Fundo, RS, no período de 1986 a 1997, em solo atualmente classificado como Latossolo Vermelho distrófico típico (Embrapa, 1999). A área experimental vinha sendo cultivada com trigo, no inverno, e com soja, no verão.

Os tratamentos foram constituídos por quatro sistemas de manejo de solo - 1) plantio direto, 2) preparo de solo com implemento cultivo mínimo (JAN) (conjunto de hastes escarificadoras + pentes de discos), no inverno, e semeadura direta, no verão, 3) preparo convencional do solo com arado de discos + grade de discos, no inverno, e semeadura direta, no verão, e 4) preparo convencional do solo com arado de aivecas + grade de discos, no inverno, e semeadura direta, no verão - e por três sistemas de rotação de culturas: sistema I (trigo/soja), sistema II (trigo/soja e ervilhaca/milho ou sorgo) e sistema III (trigo/soja, aveia-preta ou aveia-branca/soja e ervilhaca/ milho ou sorgo) (Tabela 1). As cultivares de trigo usadas foram BR-14, em 1988 e em 1989, BR-23, de 1990 a 1993 , e EMBRAPA 16, de 1994 a 1997.

Tabela 1. Sistemas de manejo de solo e de rotação de culturas em trigo. Passo Fundo, RS.

\begin{tabular}{|c|c|c|c|c|c|c|c|c|c|c|c|c|c|c|c|c|c|c|c|}
\hline \multirow{2}{*}{$\begin{array}{l}\text { Sistema de } \\
\text { rotação }\end{array}$} & \multirow{2}{*}{\multicolumn{4}{|c|}{----Parcela principal ${ }^{(1)}$---- }} & \multicolumn{15}{|c|}{ Subparcela $^{(2)}$} \\
\hline & & & & & 1986 & & & 198 & & 1989 & 19 & & 1991 & 11992 & 1993 & 1994 & $4 \quad 1995$ & 51996 & 1997 \\
\hline Sistema I & PD & PCD & PCA & PM & $\mathrm{T} / \mathrm{S}$ & $\mathrm{T} / \mathrm{S}$ & $\mathrm{T} /$ & & $\mathrm{T} / \mathrm{S}$ & & $/ \mathrm{S}$ & $\mathrm{T} / \mathrm{S}$ & & $\mathrm{T} / \mathrm{S}$ & $\mathrm{T} / \mathrm{S}$ & $\mathrm{T} / \mathrm{S}$ & $\mathrm{T} / \mathrm{S}$ & $\mathrm{T} / \mathrm{S}$ & $\mathrm{T} / \mathrm{S}$ \\
\hline \multirow[t]{2}{*}{ Sistema II } & PD & PCD & PCA & PM & $\mathrm{T} / \mathrm{S}$ & $\mathrm{E} / \mathrm{M}$ & $\mathrm{T} /$ & & $\mathrm{E} / \mathrm{M}$ & & $/ \mathrm{S}$ & $\mathrm{E} / \mathrm{N}$ & & $\mathrm{T} / \mathrm{S}$ & $\mathrm{E} / \mathrm{M}$ & $\mathrm{T} / \mathrm{S}$ & E/So & $\mathrm{T} / \mathrm{S}$ & $\mathrm{E} / \mathrm{M}$ \\
\hline & PD & PCD & PCA & PM & $\mathrm{E} / \mathrm{M}$ & $\mathrm{T} / \mathrm{S}$ & $\mathrm{E} / \mathrm{I}$ & & $\mathrm{T} / \mathrm{S}$ & & /M & $\mathrm{T} / \mathrm{S}$ & & $\mathrm{E} / \mathrm{M}$ & $\mathrm{T} / \mathrm{S}$ & E/So & $\mathrm{T} / \mathrm{S}$ & E/So & $\mathrm{T} / \mathrm{S}$ \\
\hline \multirow[t]{3}{*}{ Sistema III } & PD & PCD & PCA & PM & $\mathrm{T} / \mathrm{S}$ & $\mathrm{Ap} / \mathrm{S}$ & $\mathrm{E} / \mathrm{l}$ & & $\mathrm{T} / \mathrm{S}$ & & $/ \mathrm{M}$ & $\mathrm{Ab} /$ & & $\mathrm{T} / \mathrm{S}$ & $\mathrm{E} / \mathrm{M}$ & $\mathrm{Ab} / \mathrm{S}$ & $\mathrm{T} / \mathrm{S}$ & E/So & $\mathrm{Ab} / \mathrm{S}$ \\
\hline & PD & PCD & PCA & PM & $\mathrm{Ap} / \mathrm{S}$ & $\mathrm{E} / \mathrm{M}$ & $\mathrm{T} /$ & & $\mathrm{Ap} / \mathrm{S}$ & & $b / S$ & $\mathrm{~T} / \mathrm{S}$ & & $\mathrm{E} / \mathrm{M}$ & $\mathrm{Ab} / \mathrm{S}$ & $T / S$ & E/So & $\mathrm{Ab} / \mathrm{S}$ & $\mathrm{T} / \mathrm{S}$ \\
\hline & PD & PCD & PCA & PM & $\mathrm{E} / \mathrm{M}$ & $\mathrm{T} / \mathrm{S}$ & Ap & & $\mathrm{E} / \mathrm{M}$ & & $\mathrm{C} / \mathrm{S}$ & $\mathrm{E} / \mathrm{N}$ & & $\mathrm{Ab} / \mathrm{S}$ & $\mathrm{T} / \mathrm{S}$ & E/So & $\mathrm{Ab} / \mathrm{S}$ & $\mathrm{T} / \mathrm{S}$ & $\mathrm{E} / \mathrm{M}$ \\
\hline
\end{tabular}

(1)PD: plantio direto; PCD: preparo convencional de solo com arado de discos; PCA: preparo convencional de solo com arado de aivecas; PM: cultivo mínimo. ${ }^{(2)} \mathrm{Ab}$ : aveia-branca; Ap: aveia-preta; E: ervilhaca; M: milho; S: soja; So: sorgo; T: trigo. 
Em novembro de 1985, antes da instalação do experimento, foram realizadas a descompactação do solo com escarificador com cinzéis (Jumbo), e a correção da acidez, com 7,0 t ha ${ }^{-1}$ de calcário, de acordo com os resultados da análise de solo da área experimental. Amostras de solo, para determinação dos níveis de nutrientes e do teor de matéria orgânica, foram coletadas anualmente em todas as parcelas, após a colheita das culturas de verão.

A semeadura, o controle de plantas daninhas e os tratamentos fitossanitários foram realizados conforme recomendação para cada cultura, e a colheita de grãos foi efetuada com colhedora especial para parcelas experimentais. O rendimento de grãos de trigo foi determinado a partir da colheita de toda parcela, ajustando-se o rendimento para umidade de $13 \%$.

Foi usado o delineamento experimental de blocos ao acaso, com parcelas subdivididas e três repetições. A parcela principal foi constituída pelos sistemas de manejo de solo, e as subparcelas, pelos sistemas de rotação de culturas. A parcela principal media $360 \mathrm{~m}^{2}$ (4 m de largura por $90 \mathrm{~m}$ de comprimento), e a subparcela, $40 \mathrm{~m}^{2}$ (4 $\mathrm{m}$ de largura por $10 \mathrm{~m}$ de comprimento).

Durante o ciclo de desenvolvimento da cultura do trigo, foram realizadas quatro avaliações da incidência das manchas foliares, sendo a incidência da doença representada pelo número de folhas amostradas infectadas, expresso em porcentagem do número total de unidades avaliadas (Reis, 1994). Avaliou-se a intensidade de doenças nos estádios de afilhamento, alongamento, emborrachamento e floração. As manchas foliares foram avaliadas coletando-se, aleatoriamente, 20 plantas por parcela, as quais foram levadas ao Laboratório de Fitopatologia da Embrapa-CNPT para análise individual, tendo a incidência como critério indicador da intensidade de doença. No estádio de afilhamento, avaliaram-se todas as folhas verdes e expandidas de cada planta, e nos demais estádios, consideraram-se somente as folhas verdes expandidas da haste principal. Na maioria dos anos, as doenças predominaram nesta ordem: Stagonospora nodorum, Drechslera tritici-repentis e Bipolaris sorokiniana. Os dados originais, expressos em porcentagem de incidência, foram transformados em $(x+0,5)^{0,5}$ para análise de variância.

Procedeu-se o isolamento a partir das manchas foliares em cada época de amostragem pelo plaqueamento de 100 lesões, ao acaso, em meio de cultura, a fim de quantificar a freqüência de fungos causais de manchas foliares.

Foi efetuada análise de variância da incidência de manchas foliares (de 1991 a 1997) e do rendimento de grãos de trigo (de 1988 a 1997). Considerou-se o efeito tratamento (diferentes sistemas de manejos de solo e sistemas de rotação de culturas) como fixo, e o efeito ano, como aleatório. As médias foram comparadas entre si, pelo teste de Duncan, a 5\% de probabilidade.

\section{Resultados e Discussão}

A análise conjunta dos resultados relativos à incidência de manchas foliares de trigo (Drechsleratritici-repentis, Bipolaris sorokiniana e Stagonospora nodorum) de 1991 a 1997, no afilha-mento, no alongamento, no emborrachamento e na floração, mostrou significância do efeito de anos, da rotação de culturas, das interações ano x sistema de manejo do solo e dos sistemas de manejo de solo x rotação. Com relação à interação ano $\mathrm{x}$ sistemas de manejo de solo x rotação de culturas, houve significância somente nas duas primeiras avaliações (afilhamento e alongamento). Nos estádios mais avançados de desenvolvimento da cultura (embor-rachamento e floração), não houve efeito dos sistemas de manejo de solo e da interação ano $\mathrm{x}$ rotação de culturas. Na maioria dos anos, as doenças predominaram nesta ordem: Stagonospora nodorum, Drechslera tritici-repentis e Bipolaris sorokiniana.

A incidência de manchas foliares de trigo na maioria dos estádios avaliados foi mais elevada sob plantio direto e sob monocultura, em relação aos demais sistemas de manejo de solo e de rotação de culturas (Tabela 2). Porém, a intensidade dessas doenças sob plantio direto e rotação de culturas (sistemas II e III: 5 e $11 \%$; 11 e $11 \%$; 19 e $18 \%$; e 58 e $56 \%$, respectivamente, estádio de afilhamento, de alongamento, de emborrachamento, e de floração) foi significativamente inferior à intensidade nas plantas sob monocultura (sistema I: $23 \%, 32 \%, 33 \%$ e $56 \%$, nos mesmos estádios acima). Esses resultados foram semelhantes aos relatados por Reis (1990) e por Reis et al. (1992). Na quantificação realizada no emborrachamento, observou-se menor incidência de manchas foliares de trigo sob cultivo mínimo. Entretanto, esse tratamento foi igual, estatisticamente, ao plantio direto em relação a manchas foliares de trigo. Porém isso não se confirmou na avaliação feita na floração, em que não houve diferença entre os sistemas de manejo de solo. 
Observou-se, nas duas primeiras quantificações, que a maior incidência de manchas foliares de trigo ocorreu sob plantio direto (sistema I). Nessa situação, a emergência de trigo ocorreu junto a resíduos vegetais de culturas infectadas, o que garantiu a inoculação de esporos em plântulas desde a emergência. A explicação de não ter havido diferenças na incidência de manchas foliares no estádio de floração pode ser atribuída à taxa de infecção (R), que diminui de forma curvilinear à medida que a infecção progride (Berger, 1989; Fernandes \& Fernandez, 1991; Berger et al., 1995). De acordo com Fernandes \& Fernandez (1991), as doenças policíclicas podem completar vários ciclos de vida numa mesma planta. $\mathrm{O}$ patógeno produz nova geração de esporos a cada 7 a 10 dias. Por outro lado, a incidência de doenças foliares, em todas as avalia- ções, diminuiu com a prática da rotação de culturas por um ou dois invernos sem trigo. O aumento da incidência de manchas foliares em trigo, na monocultura, esteve relacionado diretamente com a quantidade de palha remanescente na superfície do solo. Na quantificação da palha, neste experimento, de 1993 a 1997, houve diferença significativa entre os sistemas de manejo de solo e de rotação de culturas. O plantio direto $(4,26 \mathrm{t} / \mathrm{ha})$ e o cultivo mínimo (2,50 t/ha) apresentaram valores mais elevados da quantidade de palha remanescente. Contudo, este último tratamento foi semelhante estatisticamente aos sistemas convencionais de preparo de solo com arado de discos $(0,44 \mathrm{t} / \mathrm{ha})$ e com arado de aivecas $(0,43 \mathrm{t} / \mathrm{ha})$. O plantio direto apresentou quantidade de palha maior (96\%) do que sob cultivo mínimo (48\%) e sob preparo convencional de solo com ara-

Tabela 2. Efeito de sistemas de manejo de solo e de rotação de culturas na incidência (\%) de manchas foliares de trigo, cultivar BR-23 (1991 a 1993) e cultivar EMBRAPA 16 (1994 a 1997), no estádio de afilhamento, de alongamento, de emborrachamento e de floração. Passo Fundo, $\operatorname{RS}^{(1)}$.

\begin{tabular}{|c|c|c|c|c|c|}
\hline \multirow{2}{*}{$\begin{array}{l}\text { Rotação de } \\
\text { culturas }^{(2)}\end{array}$} & \multicolumn{4}{|c|}{ Manejo de solo ${ }^{(3)}$} & \multirow[t]{2}{*}{ Média } \\
\hline & PD & PCD & PCA & PM & \\
\hline & \multicolumn{5}{|c|}{ Estádio de afilhamento (\%) } \\
\hline Sistema I & $23 \mathrm{Aa}$ & $9 \mathrm{Ba}$ & $10 \mathrm{Ba}$ & $11 \mathrm{Ba}$ & $13 \mathrm{a}$ \\
\hline Sistema II & $5 \mathrm{Ac}$ & $5 \mathrm{Aa}$ & $5 \mathrm{Ab}$ & $7 \mathrm{Ab}$ & $6 c$ \\
\hline Sistema III & $11 \mathrm{Ab}$ & $8 \mathrm{ABa}$ & $6 \mathrm{Bb}$ & $9 \mathrm{Aa}$ & $8 \mathrm{~b}$ \\
\hline \multirow[t]{2}{*}{ Média } & $13 \mathrm{~A}$ & $7 \mathrm{~B}$ & $7 \mathrm{~B}$ & 9B & 9 \\
\hline & \multicolumn{5}{|c|}{ Estádio de alongamento (\%) } \\
\hline Sistema I & $32 \mathrm{Aa}$ & $16 \mathrm{Ca}$ & $15 \mathrm{Ca}$ & $20 \mathrm{Ba}$ & $21 \mathrm{a}$ \\
\hline Sistema II & $11 \mathrm{Ab}$ & $11 \mathrm{Aa}$ & 9Аa & $10 \mathrm{Ab}$ & $10 \mathrm{~b}$ \\
\hline Sistema III & $11 \mathrm{Ab}$ & $12 \mathrm{Aa}$ & $13 \mathrm{Aa}$ & $12 \mathrm{Ab}$ & $12 \mathrm{~b}$ \\
\hline \multirow[t]{2}{*}{ Média } & $18 \mathrm{~A}$ & 13B & $12 \mathrm{~B}$ & 14B & 14 \\
\hline & \multicolumn{5}{|c|}{ Estádio de emborrachamento (\%) } \\
\hline Sistema I & $33 \mathrm{Aa}$ & $28 \mathrm{Aa}$ & $26 \mathrm{Ba}$ & $27 \mathrm{Ba}$ & $28 \mathrm{a}$ \\
\hline Sistema II & $19 \mathrm{Bb}$ & $24 \mathrm{Aa}$ & $26 \mathrm{Aa}$ & $20 \mathrm{Bb}$ & $22 \mathrm{~b}$ \\
\hline Sistema III & $18 \mathrm{Bb}$ & $23 \mathrm{Aa}$ & 26Aa & $19 \mathrm{Bb}$ & $21 \mathrm{~b}$ \\
\hline \multirow[t]{2}{*}{ Média } & $23 \mathrm{AB}$ & $25 \mathrm{~A}$ & $26 \mathrm{~A}$ & $22 \mathrm{~B}$ & 24 \\
\hline & \multicolumn{5}{|c|}{ Estádio de floração (\%) } \\
\hline Sistema I & $69 \mathrm{Aa}$ & $61 \mathrm{Ba}$ & $61 \mathrm{Ba}$ & $63 \mathrm{Ba}$ & $63 \mathrm{a}$ \\
\hline Sistema II & $58 \mathrm{Ab}$ & $60 \mathrm{Aa}$ & $62 \mathrm{Aa}$ & $58 \mathrm{Aa}$ & $59 \mathrm{~b}$ \\
\hline Sistema III & $56 \mathrm{Bb}$ & $64 \mathrm{Aa}$ & $58 \mathrm{ABa}$ & $59 \mathrm{Aa}$ & $59 \mathrm{~b}$ \\
\hline Média & $61 \mathrm{~A}$ & $62 \mathrm{~A}$ & $60 \mathrm{~A}$ & $60 \mathrm{~A}$ & 60 \\
\hline
\end{tabular}


dos de discos (8\%) e com arado de aivecas (10\%). Porém, sob rotação de culturas, a palha remanescente de trigo tende a desaparecer pela decomposição de resíduos culturais, diminuindo significativamente a incidência de manchas foliares.

A mancha amarela da folha de trigo (D. triticirepentis), cuja ocorrência aumentou com a adoção do plantio direto, começou a se destacar nesse experimento, a partir de 1989 (Santos et al., 1990). Deve-se salientar que a prevalência de determinado patógeno causador de manchas foliares é dependente da cultivar usada. A prova disso está no fato de que a mancha amarela prevaleceu na cultivar BR-23, e a septoriose, na cultivar EMBRAPA 16. Houve maior intensidade de manchas foliares de trigo no plantio direto $(25 \%)$ e no cultivo mínimo ( $24 \%$ ), em comparação com o preparo convencional de solo com arado de aivecas (21\%) e com arado de discos (17\%). Além disso, a monocultura de trigo apresentou maior incidência da doença, em comparação com um ou dois invernos de rotação. Essa diferença pode ser atribuída ao fato de o sistema de preparo de solo com arado de aivecas incorporar os resíduos culturais de trigo a maior profundidade do que os demais manejos de solo, tornando a decomposição mais rápida e, assim, eliminando a fonte de substrato para multiplicação de patógenos causadores dessas doenças. Pelas razões acima expostas, a prática do plantio direto só torna-se viável com o uso da rotação de culturas.

A análise conjunta dos resultados para rendimento de grãos de trigo (1988 a 1997) mostrou significância quanto ao efeito anos, quanto aos sistemas de manejo de solo, quanto à rotação de culturas, quanto às interações ano $\mathrm{x}$ sistemas de manejo de solo e ano x rotação de culturas. Não houve diferenças significativas no rendimento de grãos de trigo quanto às interações sistema de manejo de solo $\mathrm{x}$ rotação de culturas e ano x sistemas de manejo de solo $\mathrm{x}$ rotação de culturas. O efeito de ano indica que essas variáveis foram afetadas pela variação climática. Resultados similares de rendimento de grãos relativamente ao ano, aos sistemas de manejo de solo, à rotação de culturas, à interação de sistemas de manejo de solo x rotação de culturas e à interação ano $\mathrm{x}$ sistemas de manejo de solo $\mathrm{x}$ rotação de culturas foram relatados por Langdale et al. (1990). Nesse caso, houve aumento de rendimento de grãos, em decorrência da rotação de culturas, principalmente nas espécies de verão.

$\mathrm{O}$ rendimento de grãos de trigo foi avaliado a partir de 1988, ano em que se completou o primeiro ciclo de rotação, possibilitando observar o sistema de rotação com dois invernos sem trigo. No período de 1988 a 1997, o rendimento mais elevado ocorreu no plantio direto $\left(2.727 \mathrm{~kg} \mathrm{ha}^{-1}\right) \mathrm{e}$ no cultivo mínimo $\left(2.661 \mathrm{~kg} \mathrm{ha}^{-1}\right)$, em comparação com o de preparo convencional do solo com arado de discos $\left(2.463 \mathrm{~kg} \mathrm{ha}^{-1}\right)$ ou com arado de aivecas (2.412 $\mathrm{kg} \mathrm{ha}^{-1}$ ) (Tabela 3). Essas diferenças podem ser atribuídas à quantidade de palha remanescente, relatada anteriormente, e, como conseqüência, a deficiências hídricas em alguma fase crítica de desenvolvimento da cultura (Santos et al., 1999).

Ruedell (1995), em pesquisa na região de Cruz Alta, RS, envolvendo sistemas de manejo de solo,

Tabela 3. Efeito de sistemas de rotação de culturas no rendimento de grãos de trigo (kg ha-1) cultivar BR-14 (1988-1989), cultivar BR-23 (1990-1993), e cultivar EMBRAPA 16 (1994-1997). Passo Fundo, RS ${ }^{(1)}$.

\begin{tabular}{|c|c|c|c|c|c|}
\hline \multirow{2}{*}{$\begin{array}{l}\text { Rotação de } \\
\text { culturas }^{(2)}\end{array}$} & \multicolumn{4}{|c|}{ Manejo de solo ${ }^{(3)}$} & \multirow[t]{2}{*}{ Médic } \\
\hline & PD & PCD & PCA & PM & \\
\hline Sistema I & $2.406 \mathrm{Ac}$ & $2.091 \mathrm{Cc}$ & $2.086 \mathrm{Cc}$ & $2.230 \mathrm{Bc}$ & $2.203 \mathrm{c}$ \\
\hline Sistema II & $2.793 \mathrm{Ab}$ & $2.487 \mathrm{Bb}$ & $2.477 \mathrm{Bb}$ & $2.724 \mathrm{Ab}$ & $2.620 \mathrm{~b}$ \\
\hline Sistema III & $2.982 \mathrm{Aa}$ & $2.812 \mathrm{Ba}$ & $2.673 \mathrm{Ca}$ & $3.029 \mathrm{Aa}$ & $2.874 \mathrm{a}$ \\
\hline Média & $2.727 \mathrm{~A}$ & $2.463 \mathrm{~B}$ & $2.412 \mathrm{~B}$ & $2.661 \mathrm{~A}$ & 2.566 \\
\hline
\end{tabular}

(1)Médias seguidas da mesma letra, minúscula na vertical e maiúscula na horizontal, não apresentam diferenças significativas, a 5\% de probabilidade, pelo teste de Duncan. ${ }^{(2)}$ Sistema I: trigo/soja; sistema II: trigo/soja e ervilhaca/milho ou sorgo; sistema III: trigo/soja, aveia-preta ou aveia-branca/soja e ervilhaca/milho ou sorgo. ${ }^{(3)} \mathrm{PD}$ : plantio direto; PCD: preparo convencional de solo com arado de discos; PCA: preparo convencional de solo com arado de aivecas; PM: cultivo mínimo. 
observou que o trigo foi a espécie que sofreu menor influência do plantio direto. Dos nove anos estudados, em oito anos o plantio direto apresentou rendimento de grãos mais elevado do que o preparo convencional de solo.

O sistema de rotação de culturas com dois invernos sem trigo mostrou rendimento médio de grãos mais elevado do que sob monocultura e sob um inverno sem trigo (Tabela 3). O menor rendimento de grãos de trigo ocorreu na monocultura desse cereal. Portanto, a rotação de culturas aumenta o retorno econômico da lavoura, pelo aumento e estabilidade do rendimento de grãos, oferece alternativas de diversificação de culturas e, conseqüentemente, diminui o risco de insucesso do agricultor.

O trigo após milho ou sorgo (com um inverno de rotação) apresentou rendimento de grãos $\left(2.620 \mathrm{~kg} \mathrm{ha}^{-1}\right)$ intermediário entre a monocultura desse cereal $\left(2.203 \mathrm{~kg} \mathrm{ha}^{-1}\right)$ e a rotação de dois invernos $\left(2.874 \mathrm{~kg} \mathrm{ha}^{-1}\right)$. Esses resultados contrariam dados já obtidos por Santos et al. (1996), de 1987 a 1993, em que trigo com um inverno de rotação foi mais produtivo. Isso pode ter ocorrido devido a efeitos negativos de substâncias tóxicas decorrentes da decomposição de sorgo ou de milho (alelopatia) ou devido à falta de $\mathrm{N}$ no sistema (gramínea após gramínea).

Na Inglaterra, Slope et al. (1973) observaram que o sistema com dois invernos sem trigo apresentou maior rendimento de grãos de trigo do que a monocultura desse cereal, e, no Canadá, Sturz \& Bernier (1989) verificaram que um inverno sem trigo alternado com colza ou com linho rendeu mais que a monocultura dessa gramínea. No Estado do Paraná, Santos et al. (1996), em experimentos sob plantio direto, relataram maior rendimento de grãos nos sistemas de rotação com um inverno, dois invernos e três invernos sem trigo do que na monocultura desse cereal.

\section{Conclusões}

1. A rotação de culturas viabiliza o sistema plantio direto em relação ao controle de manchas foliares e à estabilidade do rendimento de grãos de trigo.

2. A rotação de culturas com intervalo de um inverno é suficiente para reduzir significativamente a intensidade de manchas foliares de trigo.
3. O rendimento de grãos de trigo em sistemas conservacionistas do solo é superior, quando comparado ao sistema de preparo convencional do solo.

4. O rendimento de grãos de trigo aumenta quando se utiliza a rotação de culturas com intervalo de dois invernos, em comparação com a monocultura e um intervalo de um inverno sem essa gramínea.

\section{Referências}

BERGER, R. D. Description and application of some general models for plant disease epidemics. In: LEONARD, K. J.; FRY, W. E. (Ed.). Plant disease epidemiology: genetics, resistance, and management. New York: Macmillan, 1989. v. 2, p. 15-149.

BERGER, R. D.; HAU, B.; WEBER, G. W.; BACHI, L. M. A.; BERGAMIN FILHO, A.; AMORIN, L. A simulation model to describe epidemics of rust of Phaseolus beans. I. Development of the model and sensitivity analysis. Phytopatholopgy, St. Paul, v. 85, p. 715-721, 1995.

EMBRAPA. Centro Nacional de Pesquisa de Solos (Rio de Janeiro, RJ). Sistema brasileiro de classificação de solos. Brasília: Embrapa-SPI, 1999. 412 p.

FERNANDES, J. M.; FERNANDEZ, M. R. Doenças das culturas sob manejo conservacionista. In: FERNANDES, J. M.; FERNANDEZ, M. R.; KOCHHANN, R. A.; SELLES, F.; ZENTNER, R. P. (Ed.). Manual de manejo conservacionista do solo para os estados do Rio Grande do Sul, Santa Catarina e Paraná. Passo Fundo: Embrapa-CNPT/Canadian International Development Agency, 1991. p. 53-58. (Documentos, 1).

LANGDALE, G. W.; WILSON JUNIOR, R. L.; BRUCE, R. R. Cropping frequencies to sustain long-term conservation tillage systems. Soil Science Society of America Journal, Madison, v. 54, n. 1, p. 193-198, 1990.

REES, R. G.; PLATZ, G. J. The epidemiology of yellow spot of wheat in Southern Queensland. Australian Journal of Agricultural Research, Melbourne, v. 31, n. 2, p. 259-267, 1980.

REIS, E. M. Control of diseases of small grains by rotation and management of crop residues, in Southern Brazil. In: INTERNATIONAL WORKSHOP ON CONSERVATION TILLAGE SYSTEMS, 1990, Passo Fundo. Conservation tillage for subtropical areas. Passo Fundo: Embrapa-CNPT/Canadian International Development Agency, 1990. p. 140-146. 
REIS, E. M. Importância do controle de doenças dos cereais de inverno. Correio Agrícola, São Paulo, n. 1, p. 24-27, 1998.

REIS, E. M. Manual de identificação e de quantificação de doenças do trigo. Passo Fundo: Agroalpha, 1994. 59 p.

REIS, E. M. Potencialidade de controle de doenças de trigo e de cevada por rotação de culturas. In: REUNIÃO BRASILEIRA SOBRE CONTROLE BIOLÓGICO DE DOENÇAS DE PLANTAS, 4., 1991, Campinas. Anais... Campinas: Embrapa-CNPDA, 1991. p. 78-99.

REIS, E. M.; CASA, R. T. Doenças do trigo VI: mancha amarela da folha. Passo Fundo: Bayer, 1996. 16 p.

REIS, E. M.; CASA, R. T.; BLUM, M. M. C.; SANTOS, H. P. dos; MEDEIROS, C. A. Efeitos de práticas culturais na severidade de manchas foliares do trigo e sua relação com a incidência de fungos patogênicos na semente colhida. Fitopatologia Brasileira, Brasília, v. 22, n. 3, p. 407-412, 1997.

REIS, E. M.; SANTOS, H. P. dos. Interações entre doenças de cereais de inverno e sistema plantio direto. In: EMBRAPA. Centro Nacional de Pesquisa de Trigo (Passo Fundo, RS). Plantio direto no Brasil. Passo Fundo: Embrapa-CNPT/Fundacep Fecotrigo/Fundação ABC/Aldeia Norte, 1993. p. 105-110.

REIS, E. M.; SANTOS, H. P. dos; LHAMBY, J. C. B.; BLUM, M. M. C. Effect of soil management and crop rotation on the control of leaf blotches of wheat in Southern Brazil. In: CONGRESO INTERAMERICANO DE SIEMBRA DIRECTA, 1.; JORNADAS BINACIONALES DE CERO LABRANZA, 2., 1992, Villa Giordino. Trabajos presentados... Villa Giordino: Asociación Argentina de Productores en Siembra Directa/Sociedad de Conservación de Suelos-Chile/Clube Amigos da Terra-Brasil/Fundação ABC-Brasil/ Asociación Uruguaya Pro Siembra Directa, 1992. p. 217-236.

RUEDELL, J. Plantio direto na região de Cruz Alta. Cruz Alta: Fundacep Fecotrigo, 1995. 134 p.
SANTOS, H. P. dos; LHAMBY, J. C. B.; PRESTES, A. M.; LIMA, M. R. Efeito de sistemas de manejo de solo e de rotação de culturas sobre o rendimento de grãos de trigo. In: REUNIÃO NACIONAL DE PESQUISA DE TRIGO, 18., 1999, Passo Fundo. Anais... Passo Fundo: Embrapa-CNPT, 1999. v. 2, p. 691-698.

SANTOS, H. P. dos; REIS, E. M.; DERPSCH, R. Rotação de culturas. In: EMBRAPA. Centro Nacional de Pesquisa de Trigo (Passo Fundo, RS). Plantio direto no Brasil. Passo Fundo: Embrapa-CNPT/Fundacep Fecotrigo/ Fundação ABC/Aldeia Norte, 1993. p. 85-103.

SANTOS, H. P. dos; REIS, E. M.; LHAMBY, J. C. B.; PEREIRA, L. R.; ROMAN, E. S.; SALVADORI, J. R. Efeito do manejo do solo e de sistemas de cultivo sobre a produção de trigo. Passo Fundo: Embrapa-CNPT, 1990. 15 p. Programa Nacional de Pesquisa - Trigo, projeto 004.86.00816.

SANTOS, H. P. dos; REIS, E. M.; LHAMBY, J. C. B.; WOBETO, C. Efeito da rotação de culturas sobre o trigo, em sistema plantio direto, em Guarapuava, PR. Pesquisa Agropecuária Brasileira, Brasília, v. 31, n. 4, p. 259-267, abr. 1996.

SLOPE, D. B.; ETHERIDGE, J.; WILLIAMS, R. J. B. Grain yield and incidence of take-all and eyespot in winter wheat grown in different crop sequences at Saxmundham. Rothamsted Experimental Station Report for 1972, Harpenden, pt. 2, p. 160-167, 1973.

STURZ, A. V.; BERNIER, C. C. Influence of crop rotations on winter wheat growth and yield in relation to the dynamics of pathogenic crown and root rot fungal complexes. Canadian Journal of Plant Pathology, Guelph, v. 11, n. 1, p. 114-121, 1989.

SUTTON, J. C.; VYN, T. J. Crop sequences and tillage practices in relation to diseases of winter in Ontario. Canadian Journal of Plant Pathology, Guelph, v. 12, n. 4, p. 358-368, 1990.

WIESE, W. V. Compendium of wheat diseases. St. Paul: American Phytophatological Society, 1987. 112 p. 